\title{
An Improved Social Force Model for Bicycle Flow in Groups
}

\author{
Ying-Xu Rui $\mathbb{D}{ }^{1}$ Tie-Qiao Tang $\mathbb{D}^{1}$, and Jian Zhang $\mathbb{D}^{2}$ \\ ${ }^{1}$ School of Transportation Science and Engineering, \\ Beijing Key Laboratory for Cooperative Vehicle Infrastructure Systems and Safety Control, Beihang University, \\ Beijing 100191, China \\ ${ }^{2}$ Beijing Key Laboratory of Traffic Engineering, Beijing University of Technology, Beijing 100124, China
}

Correspondence should be addressed to Tie-Qiao Tang; tieqiaotang@buaa.edu.cn

Received 7 August 2021; Revised 9 September 2021; Accepted 9 September 2021; Published 18 September 2021

Academic Editor: Yuan Gao

Copyright (C) 2021 Ying-Xu Rui et al. This is an open access article distributed under the Creative Commons Attribution License, which permits unrestricted use, distribution, and reproduction in any medium, provided the original work is properly cited.

Bicycle flow widely has group behavior (i.e., cyclists have a tendency to ride in groups), which may have some significant effects on the bicycle's motion. However, the existing studies on bicycle flow rarely consider this factor. Generally, bicycle flow has two kinds of group behaviors, i.e., shoulder group behavior and following group behavior. In this paper, we propose an improved social force (SF) model to describe the two kinds of group behaviors. Then, we use the improved SF model to, respectively, explore the effects of the two kinds of group behaviors on the bicycle's motion from the simulation perspective. The numerical results show that (i) shoulder group behavior has some negative impacts on the bicycle's motion, i.e., the critical density (where the through capacity can reach the maximum value), the jam density, and the through capacity will be reduced; (ii) following group behavior has some positive impacts on the bicycle's motion, i.e., the critical density, the jam density, and the through capacity will be enhanced; (iii) the impacts of coexistence of shoulder and following group behavior are related to the density. Besides, increasing group size and group probability will enlarge the negative impacts of shoulder group behavior and alleviate the positive impacts of following group behavior. These results can guide administrators to better manage bicycle flow (especially reasonably control the negative impacts of group behaviors).

\section{Introduction}

Due to the bicycle's own merits (i.e., low price and no emissions), bicycle has been an important commuting tool in many countries [1], and cyclists have a tendency to ride in groups (especially after shared bicycles occur) [2]. For instance, if a family does a short distant trip in cities, the family members will often select shared bicycles to ride in a group, i.e., group behavior occurs at this time. Generally speaking, the bicycle flow has two kinds of group behaviors, i.e., shoulder group behavior and following group behavior. The two kinds of group behavior widely exist in an urban traffic system (see Figure 1), frequently lead to inner interactions among bicycles, and may significantly impact the whole traffic system $[3,4]$. However, little effort has been made to study the impacts. Therefore, it is essential to propose one precise motion model accounting for group behaviors to explore the complex phenomena resulting from the bicycle flow in groups.

For bicycle flow, the microscopic model is suitable for studying the microcosmic phenomena and inner interactions among bicycles. Generally speaking, the existing microscopic models for bicycle flow can roughly be divided into cellular automaton (CA) models and social force (SF) models. Due to the similarities between bicycle flow and vehicle flow, researchers extended the CA model for vehicle flow [5] to explore the bicycle's motion, including lanechanging, passing, lateral dispersion, and retrograde behavior [6-10]. The CA model has some extensible rules and can reproduce each bicycle's microbehavior. However, limited by the discrete motion, this model cannot accurately describe the interactions among bicycles (especially compared with the continuous model).

To overcome this drawback, researchers extended the SF model for pedestrian flow $[11,12]$ to describe the bicycle's 


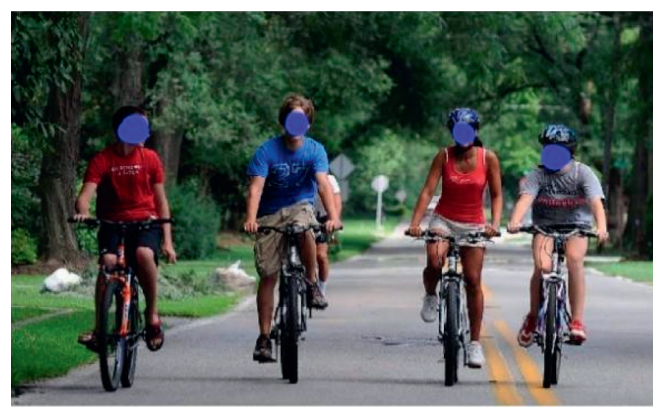

(a)

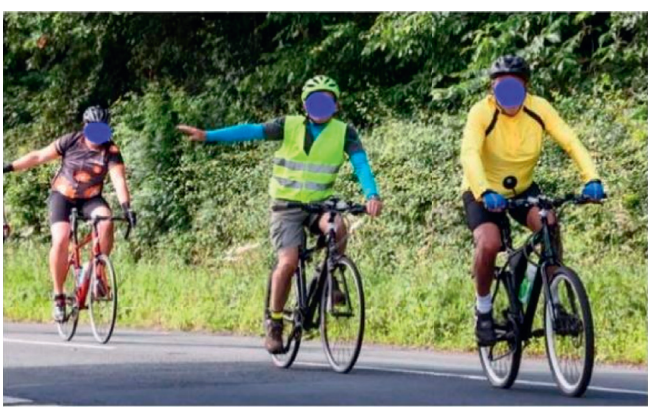

(b)

Figure 1: Bicycle's two typical group behaviors. (a) Shoulder group behavior and (b) following group behavior.

motion [13-17]. The SF model uses a physics concept, social force $[18,19]$, to describe the interaction among bicycles. Unlike the CA model, the interaction among bicycles in the SF model is described by social forces formulated by continuous variables, so the SF model is more suitable to accurately reproduce the interactions among bicycles. The relevant parameters are required to be calibrated regardless of the excellent simulation performance. On this issue, Zhao and Zhang [16] used experimental data to respectively calibrate the speed-density functions of vehicle flow, bicycle flow, and pedestrian flow and then developed a unified SF model for different kinds of traffic flow. However, the unified SF model hardly describes the route choice and conflict avoidance of bicycles at intersections. Thus, researchers embedded one multilayered process framework in the SF model to simulate bicycles' complex motion at intersections. For instance, Gavriilidou et al. [20] designed a two-layered microscopic model to reproduce the formation of bicycle queue, where the SF model is proposed in the second layer to determine a feasible trajectory. Li et al. [21] embedded the decision process in the SF model and designed a behavior force to explore the lateral dispersion phenomenon of high density through bicycle flow at an intersection.

Although researchers have done fruitful work on bicycle flow, the above SF models cannot be used to study the impacts of group behaviors on bicycle flow directly since they did not consider this factor. To study the bicycle group behavior, Tang et al. [22, 23] proposed an extended CA model to evaluate the impacts of shoulder group behavior and following group behavior on bicycle flow in two typical traffic scenarios, but they did not analyze the impacts from the perspective of continuous model, such as SF model. In this paper, we develop an improved SF model to explore the impacts of shoulder group behavior and following group behavior on bicycle flow. The rest of this paper is organized as follows: an improved SF model accounting for group behavior is proposed in Section 2; numerical tests are carried out to evaluate the impacts of group behavior on bicycle flow in Section 3; and some conclusions are summarized in Section 4.

\section{Extended SF Model Accounting for Group Behavior}

In this section, we propose an extended SF model accounting for group behavior based on the unified SF model [16]. To better depict the motion of bicycles in groups, we should give the following basic assumptions:

(1) The scenario is defined as Figure 2, where the road is channelized as three lanes, and two bicycles cannot move abreast on each lane [16].

(2) The bicycles are divided into three categories, i.e., with shoulder group behavior, with following group behavior, and without group behavior. Each type of bicycles and riders is homogeneous.

(3) Lane-changing and retrograde behavior are both prohibited (given that bicycles with group behavior tend to keep groups stable, and with reference to the work of Zhao and Zhang [16], we can assume that lane-changing is prohibited).

(4) The impacts of road/lane border on each bicycle motion are ignored.

(5) The bicycles without shoulder group behavior are not affected by the ones on their adjacent lanes.

As for the SF model for bicycle flow, Zhao and Zhang [16] pointed out that three forces should be used to depict each bicycle's motion, i.e., the driving force, the interaction forces from adjacent bicycles, and the interaction forces from road boundaries. Thus, they proposed one SF model for bicycle flow, i.e.,

$$
m_{i} * \frac{\mathrm{d} v_{i}}{\mathrm{~d} t}=f_{i d}+\sum_{j(j \neq i)} f_{i j}+\sum_{w} f_{i w}
$$

where $m_{i}, v_{i}, f_{i d}$ are, respectively, the ith bicycle's mass, speed, and driving force, $f_{i j}$ is the interaction force resulting from the $j$ th bicycle, and $f_{i w}$ is the interaction force resulting from the road boundary $w$. As for the three forces $f_{i d}, f_{i j}, f_{i w}$, Zhao and Zhang defined them as follows [16]: 


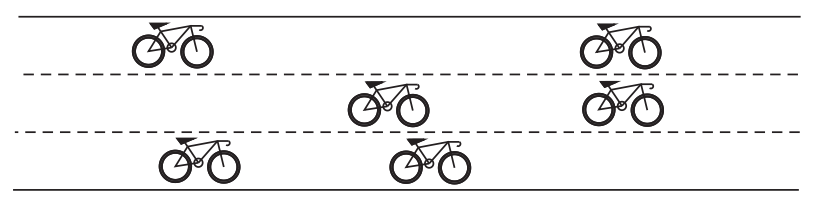

ridding direction

FIgURE 2: The simulation scenario of a three-lane circuit road, where the bicycle may have two kinds of group behaviors.

$$
\begin{aligned}
& f_{i d}=m_{i} * \frac{v_{i}^{0}-v_{i}}{\tau}, \\
& v_{i}^{0}= \begin{cases}0, & d_{i} \leq d_{\text {safe }}, \\
k *\left(d_{i}-d_{\text {safe }}\right), & d_{\text {safe }}<d_{i} \leq d_{\text {safe }}+\frac{v_{\text {max }}}{k}, \\
v_{\max }, & d_{i}>d_{\text {safe }}+\frac{v_{\text {max }}}{k}, \\
f_{i j}= & A * \exp \left(\frac{r_{i j}-d_{i j}}{B}\right) * e_{i j}, \\
f_{i w}= & A * \exp \left(\frac{r_{i}-d_{i w}}{B}\right) * e_{i w},\end{cases}
\end{aligned}
$$

where $v_{i}^{0}$ is the $i$ th bicycle's desired speed, $\tau$ is the relaxation time, $d_{\text {safe }}, v_{\text {max }}$ are, respectively, the minimum safety distance and maximum speed, $d_{i}$ is the distance between the $i$ th bicycle and the preceding one, $r_{i j}$ is the sum of the $i$ th bicycle's radius and the $j$ th bicycle's radius, $d_{i j}$ is the center distance between the $i$ th bicycle and the $j$ th bicycle, $e_{i j}$ is the unit vector pointing from the $j$ th bicycle to the $i$ th bicycle, $r_{i}$ is the radius of the $i$ th bicycle, $d_{i w}$ is the center distance between the $i$ th bicycle and the road boundary $w, e_{i w}$ is the unit vector pointing from the border $w$ to the $i$ th bicycle, and $k, A, B$ are constants.

However, the above SF model cannot reproduce the impacts of group behavior on each bicycle's motion since it does not consider this factor. Shoulder group behavior and following group behavior widely exist, so we should respectively incorporate the two kinds of group behaviors into the SF model [16]. Before exploring this topic, we should give the definitions of the two group behaviors as follows.

Definition 1. Shoulder group behavior is a tendency to make the difference between longitudinal positions of arbitrary two bicycles in one shoulder group as small as possible (see Figures 3(a) and 3(b)).

Definition 2. Following group behavior is a tendency to keep the difference between the longitudinal positions of the current bicycle and its preceding/following bicycles as a fixed value (see Figures 4(a) and 4(b)).

First, we embed shoulder group behavior in the SF model. In this developed SF model, an attractive force among the bicycles with shoulder group behavior makes the difference between the headway of arbitrary two/three bicycles as small as possible to ensure that these bicycles are in the same horizontal position. In this paper, we call the attractive force "shoulder group force." Based on the above discussion, we can simply define shoulder group force as follows:

$$
\begin{aligned}
f_{g}^{s} & =\sum_{n=1}^{K-1} C *\left[\exp \left(\frac{d_{i n}}{D}\right)-1\right] * e_{i n}+m_{i} * \frac{v_{i}^{\min }-v_{i}^{0}}{\tau}, \\
v_{i}^{\min } & =\min \left\{v_{1}^{0}, v_{2}^{0}, v_{3}^{0}, \ldots, v_{K}^{0}\right\},
\end{aligned}
$$

where $C, D$ are constants, $d_{\text {in }}$ is the longitudinal center distance between the $i$ th bicycle and its adjacent member $n$ in the group, $e_{i n}$ is the unit vector pointing from the $n$th bicycle to the $i$ th bicycle, $K$ is the group size, $v_{i}^{\min }$ is the minimum desired speed of the bicycles in the group, and $\left\{v_{1}^{0}, v_{2}^{0}, v_{3}^{0}, \ldots, v_{K}^{0}\right\}$ is the set of the desired speed in the group. The first item of equation (3) is the resultant force of several forces that other bicycles in the group exert on the $i$ th bicycle; and the second item of equation (3) is a correction term of driving force to make bicycles in the group share the same least desired speed.

Next, we embed the following group behavior in the SF model. For each following group, the bicycles can be divided into one leader and several followers. In this developed SF model, an attractive force in the group makes the longitudinal distance between each follower and its preceding one approximately equal to their desired distance. In this paper, we call the attractive force "following group force." Based on the above discussions, we can simply define the following group force as follows:

$$
\begin{aligned}
& f_{g}^{f}=C *\left[\exp \left(\frac{d_{i m}-d_{f}}{D}\right)-1\right] * e_{i m} \\
& d_{f}=\eta * d_{\text {safe }}
\end{aligned}
$$

where $d_{i m}$ is the center distance between the $i$ th bicycle and its adjacent one $m$ in the group on the same lane, $e_{i m}$ is the unit vector pointing from the $m$ th bicycle to the $i$ th bicycle, $d_{f}$ is the following distance, $\eta$ is a scaling factor, and $d_{\text {safe }}$ is the minimum safe distance of the bicycles without group behavior. The right side of equation (5) is the attractive force that the $m$ th bicycle exerts on the $i$ th bicycle.

Based on the above discussions, we can embed shoulder/ following group force into the SF model for bicycle flow [16]. Thus, we can propose an extended SF model for bicycle flow with group behavior, i.e.,

$$
m_{i} \frac{\mathrm{d} v_{i}}{\mathrm{~d} t}=f_{d}+\sum_{j(j \neq i)} f_{i j}+\sum_{w} f_{i w}+f_{g} .
$$

Comparing with the SF model [16], the extended SF model has explicitly considered shoulder/following group force $f_{g}$. Therefore, the extended SF model can directly be used to study the impacts of group behaviors on bicycle flow. For convenience, we can unify the group force $f_{g}$ as follows: 


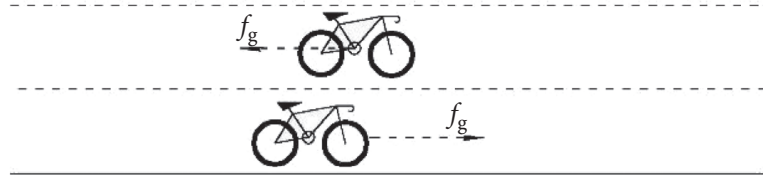

(a)

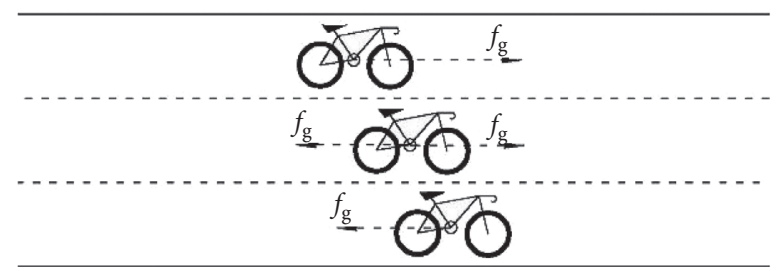

(b)

FIgURE 3: The schematic diagrams of shoulder group force. The group sizes in (a) and (b) are, respectively, two and three.

$$
f_{g}= \begin{cases}\sum_{n=1}^{K-1} C *\left[\exp \left(\frac{d_{i n}}{D}\right)-1\right] * e_{i n}+m_{i} * \frac{v_{i}^{\min }-v_{i}^{0}}{\tau}, & b_{i} \in B_{N_{1}}, \\ C *\left[\exp \left(\frac{d_{i m}-d_{f}}{D}\right)-1\right] * e_{i m}, & b_{i} \in B_{N_{2}}, \\ 0, & b_{i} \in B_{N_{3}},\end{cases}
$$

where $b_{i}$ represents the $i$ th bicycle and $B_{N_{1}}, B_{N_{2}}, B_{N_{3}}$ are the sets of the bicycles with shoulder group behavior, with following group behavior, and without group behavior.

\section{Numerical Tests}

In this section, we use the proposed model to study the impacts of the two kinds of group behaviors on each bicycle's movement and bicycle flow. Before studying the impacts, we define the simulation scenario and other related simulation conditions as follows:

(a) The simulation scenario is set as a three-lane circuit road, where the road length $L$ is equal to $49 \mathrm{~m}$, and the road width $W$ is equal to $2 \mathrm{~m}$ [16]

(b) A fixed number of bicycles are uniformly distributed on each lane of the road, where the numbers of bicycles, $N$, are, respectively, set as 15, 19, and 23 in the following simulations [16]

(c) The bicycles with group behavior are randomly distributed, where the group probability $p_{\text {group }}$ is beforehand defined as a constant

(d) Other parameters of the proposed model are listed in Table 1, where the values of some parameters are set as those calibrated by Zhao and Zhang [16], and those of other parameters are defined in this paper

First, we use the proposed model to simulate each bicycle's trajectory under the following four cases:

Case I: each bicycle does not have group behavior

Case II: only some bicycles have shoulder group behavior

Case III: only some bicycles have following group behavior

Case IV: shoulder group behavior and following group behavior coexist in the three-lane system
Here, we focus on studying the effects of shoulder group behavior and following group behavior on each bicycle's trajectory, where the group size and the group probability are, respectively, set as 2 and 0.5 in Case II, Case III, and Case IV, and the bicycles with group behavior are stochastically distributed in the three-lane system.

Figures 5-8, respectively, list each bicycle's trajectory in Case I, Case II, Case III, and Case IV under different $N$. The three-lane systems in Case I and Case III can both be regarded as three independent ones due to the following reasons:

(i) Lane-changing behavior is prohibited

(ii) The number of bicycles on each lane is identical

(iii) The distribution of bicycles with group behavior on each lane is the same in Case III

Therefore, Figures 5 and 7 only list each bicycle's trajectory on the middle lane. However, in Case II and Case IV, the bicycles with shoulder group behavior may affect the motions of other bicycles on the adjacent lanes, so we should provide each bicycle's trajectory on each lane in Figures 6 and 8. In each subfigure of Figures 5-8, the horizontal coordinate is time, the vertical coordinate is spatial position, and the instantaneous speed $v_{i}(t)$ is exhibited by different colors. From Figure 5, we can conclude the following findings:

(1) In Figure 5(a), the bicycle flow is approximately free flow at the early stage and then evolves into a stopand-go flow

(2) In Figures 5(b) and 5(c), the initial velocity is relatively low, and the bicycle flow quickly evolves into a stop-and-go flow

(3) When the density increases, the bicycle's average headway and speed both decrease

In Case II, the group size is set as 2 , so the two bicycles with group behavior exist on the left lane and the middle lane (or on the middle lane and the right lane); i.e., the number of bicycles with group behavior on the middle lane is just equal to the sum of those on the left lane and the right lane. At this time, shoulder group behavior has greater impacts on the bicycle flow on the middle lane than those on the left and right lanes (see Figure 6). In addition, from Figure 6, we can conclude the following findings:

(1) In Figures 6(a)-6(c), the backward wave on the middle lane is more frequent than that on the left 


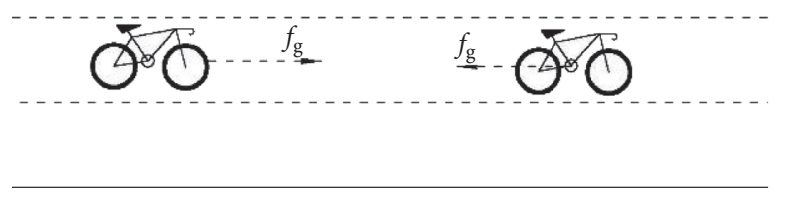

(a)

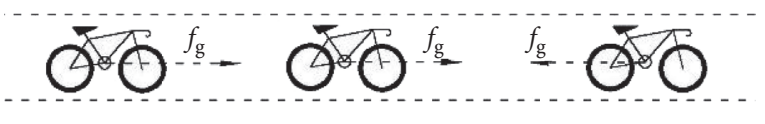

(b)

Figure 4: The schematic diagrams of following group force. The group sizes in (a) and (b) are, respectively, two and three.

TABLE 1: The related parameters of the extended SF model.

\begin{tabular}{lccc}
\hline Parameters & Values & Parameters & Values \\
\hline$v_{\max }(\mathrm{m} / \mathrm{s})$ & $N\left(3.50,0.65^{2}\right)$ & $m(\mathrm{~kg})$ & 90 \\
$k\left(\mathrm{~s}^{-1}\right)$ & $N\left(1.42,0.16^{2}\right)$ & $\tau$ & 0.5 \\
$d_{\min }(m)$ & $N\left(2.08,0.38^{2}\right)$ & $\eta$ & 0.75 \\
$A(\mathrm{~N})$ & 500 & $C(\mathrm{~N})$ & 500 \\
$B(\mathrm{~m})$ & 0.08 & $D(\mathrm{~m})$ & 100 \\
$r(\mathrm{~m})$ & 0.78 & & \\
\hline
\end{tabular}

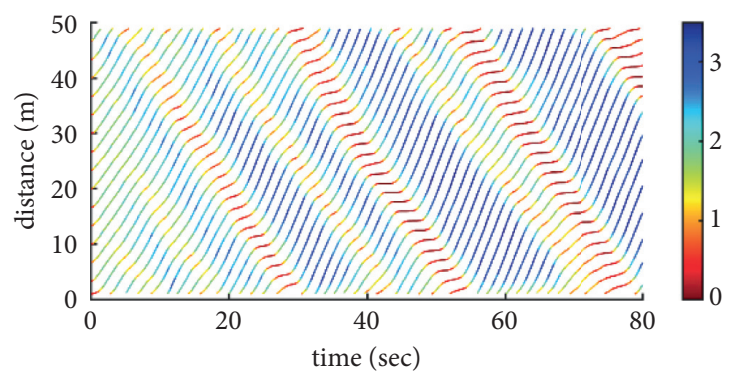

(a)

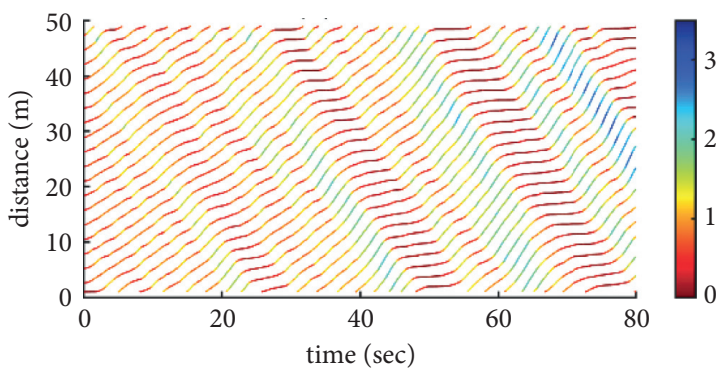

(b)

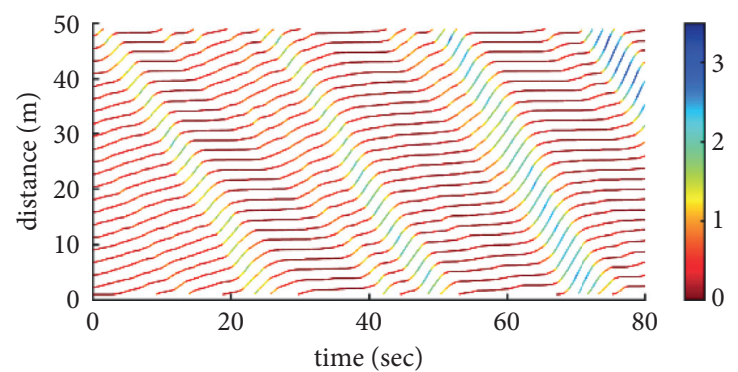

(c)

Figure 5: Each bicycle's trajectory on the middle lane in Case I where the fixed numbers of bicycles on each lane in (a), (b), and (c) are, respectively, 15,19 , and 23 . (a) $N=15$. (b) $N=19$. (c) $N=23$.

lane and right lane, but the difference will decrease with the increase of density (see Figures 6(d)-6(f) and Figures 6(g)-6(i)).

(2) When the density is low or moderate (i.e., $N=15$ or 19), some bicycles will constantly maintain a relatively high headway, where the headway may vary but is much higher than that of other bicycles (see Figures 6(a)-6(f)).

(3) When the bicycle density is high (i.e., $N=23$ ), there are no differences in the corresponding subfigures, which shows that the effect of group behavior on bicycle flow will decrease with the increase of density.

(4) Comparing with each subfigure of Figure 5, the bicycle flow in the corresponding subfigure in Figure 6 is less stable, but the speed is slightly lower, and the congestion is slightly more serious, which indicates that shoulder group behavior has some negative impacts on bicycle flow. This conclusion is consistent with that of shoulder group behavior based on the CA model [22]. Hence, if possible, shoulder group behavior should be prohibited. 


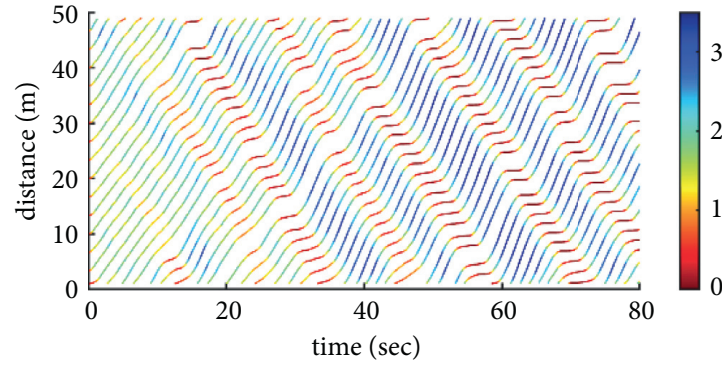

(a)

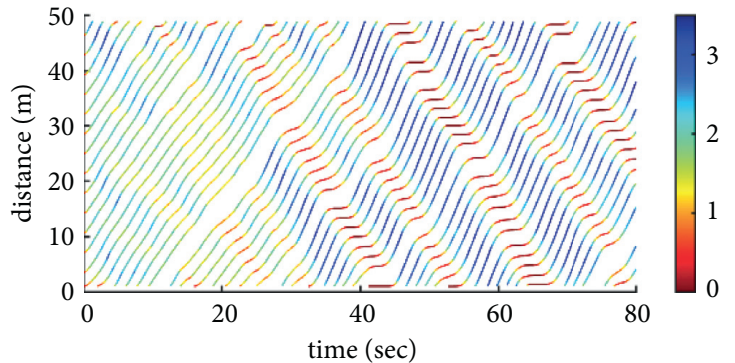

(c)

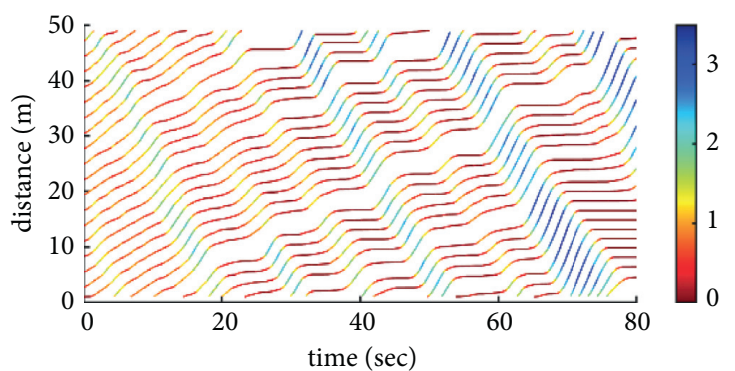

(e)

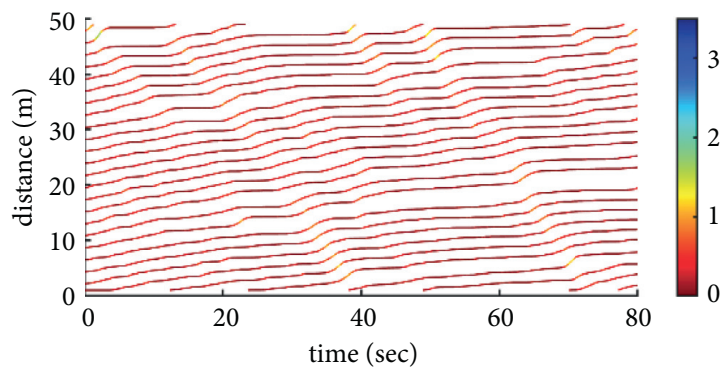

(g)

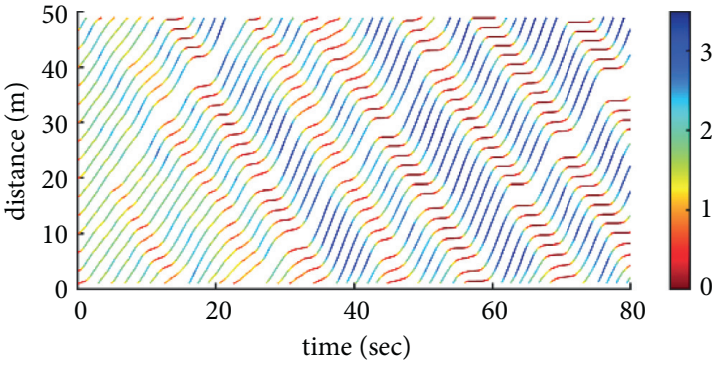

(b)

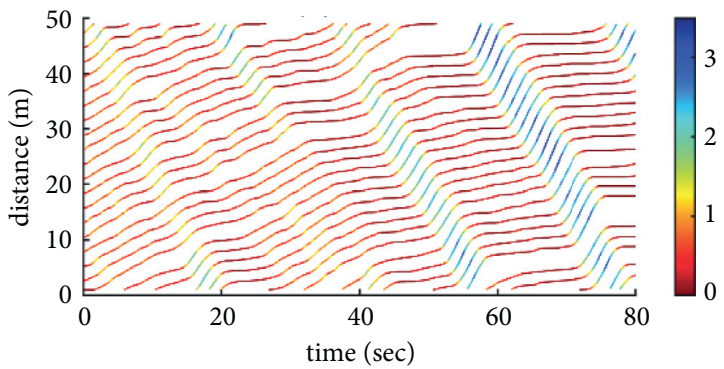

(d)

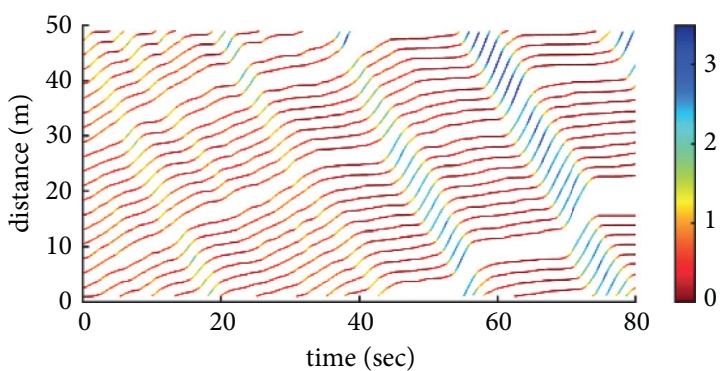

(f)

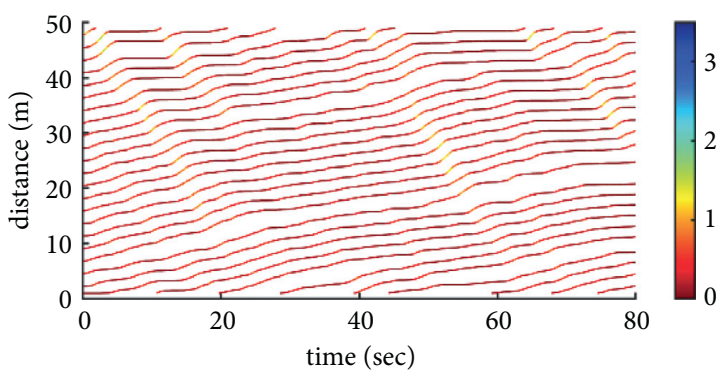

(h)

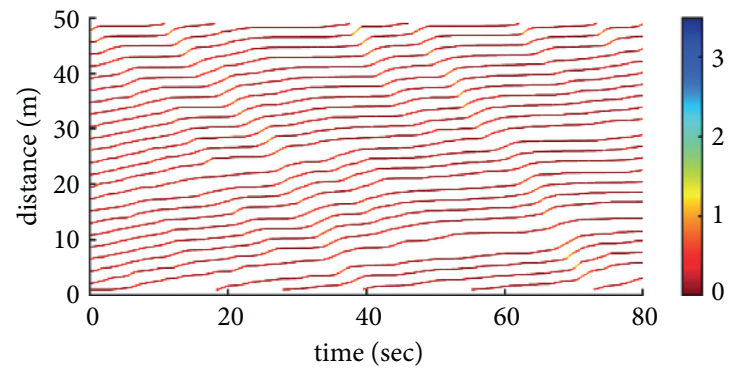

(i)

Figure 6: Each bicycle's trajectory in Case II under different $N$. (a-c) Left lane, (d-f) middle lane, and (g-i) right lane. (a) $N=15$. (b) $N=15$. (c) $N=15$. (d) $N=19$. (e) $N=19$. (f) $N=19$. (g) $N=23$. (h) $N=23$. (i) $N=23$. 
In Case III, the follower in each group often keeps a relatively small headway, so following group behavior may have some positive effects on bicycle flow (see Figure 7), where the detailed conclusions can be summarized as follows:

(1) The bicycle flow is approximately free flow and always keeps the smooth state, which shows that the stability of bicycle flow in Case III is higher than the ones in the other cases.

(2) When the density increases, the bicycle's average speed decreases, but neither stop-and-go nor congestion occurs.

(3) Comparing with each subfigure of Figures 5 and 6 , the bicycle flow in Figure 7 has a higher average speed, lighter congestion, and better stability, which shows that following group behavior has some positive effects on bicycle flow. This conclusion is also consistent with that of following group behavior based on the CA model [22].

In Case IV, the bicycles with shoulder group behaviors and the bicycles with following group behavior are mixed in all bicycles. Unlike the above three cases, the group probabilities of shoulder and following group behaviors are both set as 0.25 , and the total group probability equals 0.5 . As mentioned earlier, shoulder group behavior has some negative impacts on the bicycle's motion, and following group behavior has some positive impacts on the bicycle's motion. Therefore, the bicycle motion in Case IV is better than that in Case II and worse than that in Case III (see Figure 8). In addition, we can conclude the following findings:

(1) When the density is low (i.e., $N=15$ ), the negative impacts of shoulder group behavior are less than the positive impacts of following group behavior. This can be proved by the phenomenon that the bicycle flow in Figures 8(a)-8(c) is constantly free flow and is prominently better than corresponding subfigures in Case I and Case II.

(2) When the density is moderate or high (i.e., $N=19$ or 23), the negative impacts of shoulder group behavior are greater than the positive impacts of following group behavior. This can be elucidated by the phenomenon that the bicycle flow in Figures $8(\mathrm{~d})-8(\mathrm{i})$ is congested and prominently worse than that in the corresponding subfigures in Case I and Case III.

(3) The above findings show that shoulder group behavior should better be prohibited (especially under moderate/high density).

The above simulation results indicate that shoulder group behavior and following group behavior both change the bicycle's motion, i.e., impact the speed and headway from the microperspective. These microchanges inevitably lead to some changes in macro bicycle flow characteristics. To study the impacts, we should explore the fundamental diagrams under different group behaviors. Before studying this topic, we should give the following notes:

(i) To reduce the impacts of initial conditions on the simulation results, the flow, speed, and density are calculated by use of the simulation data after 50 seconds (considering that the first 50 seconds of the numerical test is an initialization process, the effect of group behavior on bicycle flow has not been demonstrated yet, and we can ignore this part of the data).

(ii) To reduce randomness, numerous numerical tests are carried out to calculate the average value of each statistical indicator.

(iii) Using the method in the study [16], the density $\rho(t)$, the speed $v(t)$, and the flow $J(t)$ at time $t$ can be calculated by the following equations:

$$
\begin{aligned}
& \rho(t)=\frac{\sum_{i=1}^{N_{t}} \theta_{i}(t)}{l_{m}}, \\
& \theta_{i}(t)=\frac{l_{i}}{d_{i}}, \\
& v(t)=\frac{\sum_{i=1}^{N_{t}} v_{i}(t)}{N_{t}}, \\
& J(t)=\rho(t) * v(t),
\end{aligned}
$$

where $N_{t}$ is the number of bicycles in the tested area at time $t, l_{m}$ is the length of the tested area, $\theta_{i}(t)$ is the contribution of the $i$ th bicycle to the density, $l_{i}$ is the overlapping length between the ith bicycle's headway and the tested area, and $v_{i}(t)$ is the $i$ th bicycle's velocity at time $t$.

To better display the impacts of group behavior on the fundamental diagram, we fit the speed data calculated by the proposed SF model into a unified curve, which can be described by the Newell model [24]. However, since the critical density calculated by equation (9) is always higher than the one in the Newell model, we here modify the speed-density function [24] as follows:

$$
v(\rho)=V_{f} *\left(1-\exp \left(-\frac{\mu}{V_{f}} *\left(\frac{1}{\rho-\rho_{0}}-\frac{1}{\rho_{j}-\rho_{0}}\right)\right)\right),
$$

where $v(\rho)$ is the speed defined by density, $V_{f}$ is the free flow speed, $\rho$ is the density, $\rho_{j}$ is the jam density, $\rho_{0}$ is the critical density and it equals $155 \mathrm{bic} / \mathrm{km}$, and $\mu$ is a constant.

Based on the above discussions, we can apply the simulation data to calibrate the related parameters in equation (10) (see Table 2).

Next, we study the impacts of group behavior on the fundamental diagram, where the group probability is set as 0.5 . Figure 9 displays the speed-density and flow-density relationships under Cases I, II, and III. Figures 10 and 11 display the speed-density and flow-density relationships under different group sizes (i.e., $K=1,2,3$ ). In Figures 9-11, 


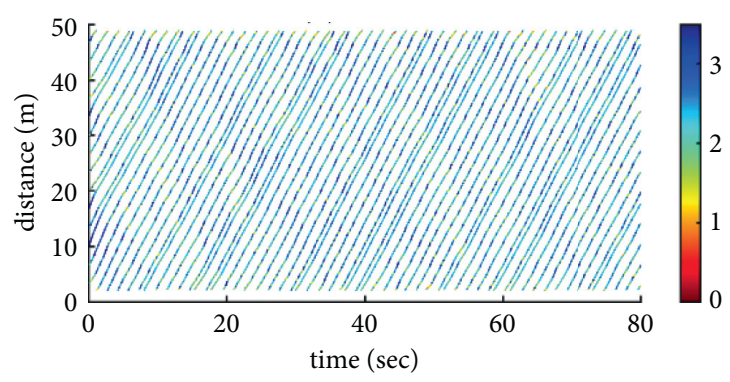

(a)

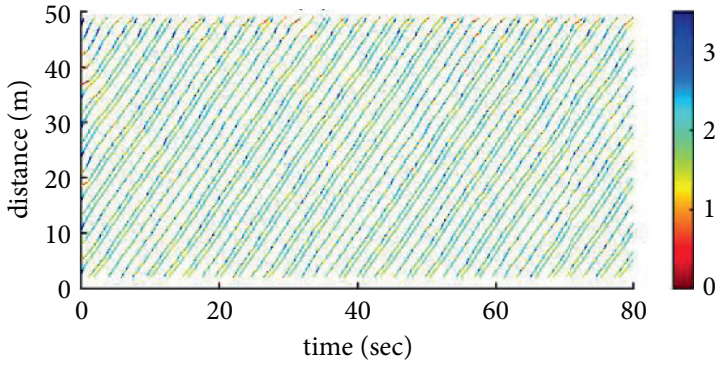

(b)

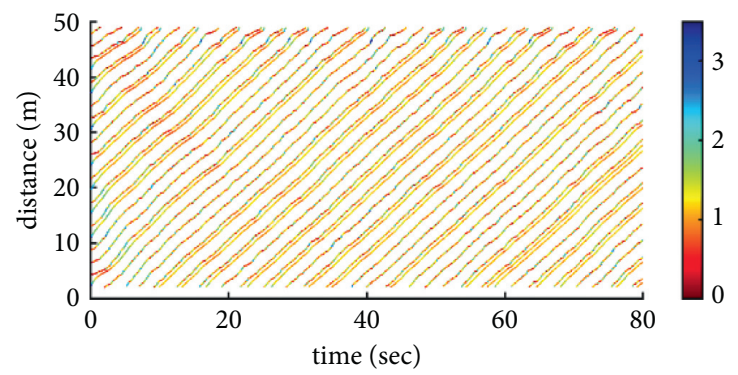

(c)

FiguRE 7: Each bicycle's trajectory on the middle lane in Case III, where the fixed numbers of bicycles on each lane in (a), (b), and (c) are, respectively, 15,19 , and 23 . (a) $N=15$. (b) $N=19$. (c) $N=23$.

the horizontal coordinate is speed or flow, the vertical coordinate is density, the scattered data are the ones that are calculated by the proposed model, and the curves are the ones that are fitted by equation (10). From Figure 9, we can conclude the following findings:

(1) When the density is very low (i.e., $\rho<250 \mathrm{bic} / \mathrm{km}$ ), there are no differences between the three cases, which indicates that group behavior has no impacts on bicycle flow.

(2) When the density is relatively high (i.e., $\rho \geq 250 \mathrm{bic} / \mathrm{km}$ ) and increases, the speed drops, and the flow first increases and then drops, which just shows that the two kinds of group behaviors do not change the qualitative features of the fundamental diagram, but there are quantitative differences in the three cases; i.e., the speed and the flow are the highest in Case III but the lowest in Case II. In addition, the critical density (where the through capacity can reach the maximum value), the jam density, and the maximum flow are the lowest in Case II but the highest in Case III, which shows that shoulder group behavior has some negative impacts on the speed and the flow while following group behavior has some positive impacts on the speed and the flow.

From Figure 10, we can conclude the following findings:

(1) When $\rho<250 \mathrm{bic} / \mathrm{km}$, shoulder group behavior has no prominent effects on the speed and flow under $K=2$, but it prominently reduces the speed and flow under $K=3$. In addition, some scattered yellow dots appear at the bottom left of the yellow curve, which shows that even if the density is relatively small, the bicycle flow under $K=3$ is unstable and congested.

(2) When $\rho \geq 250 \mathrm{bic} / \mathrm{km}$, the speed and flow are both the highest under $K=1$ but the least under $K=3$. In addition, the critical density, the jam density, and the maximum flow decrease with the group size. The results show that the negative impacts of shoulder group behavior on bicycle flow will become more prominent with the increase of group size.

From Figure 11, we can conclude the following findings:

(1) When $\rho<250 \mathrm{bic} / \mathrm{km}$, following group behavior has no prominent impacts on the speed and flow under $K=2$, while it slightly reduces the speed and flow under $K=3$. In addition, some scattered yellow dots appear at the bottom and top of the yellow curve, which shows that even if the density is relatively small, the bicycle flow under $K=3$ is unstable.

(2) When $\rho \geq 250 \mathrm{bic} / \mathrm{km}$, the speed and flow are both the highest under $K=2$ and the lowest under $K=1$; the critical density, the jam density, and the maximum flow increase when the group size switches from 1 to 2 and decrease when the group size switches from 2 to 3 . These results indicate that following group behavior has some positive impacts on bicycle flow, but the positive impacts will be alleviated with the increase of group size.

The above simulation results indicate that shoulder group behavior and following group behavior have some impacts on bicycle flow under the fixed $p_{\text {group }}$, where the impacts are related to both the density and the group size. In fact, the 


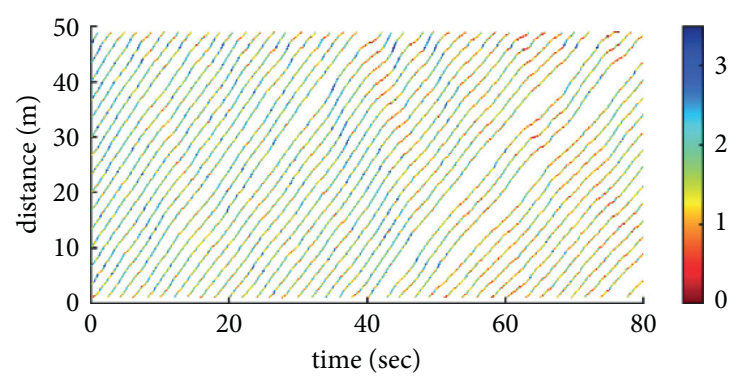

(a)

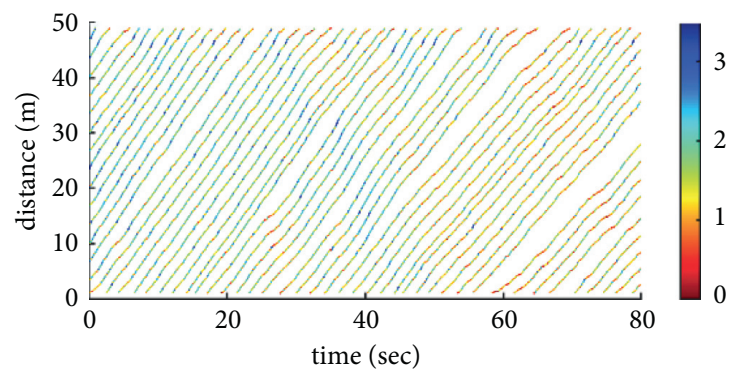

(c)

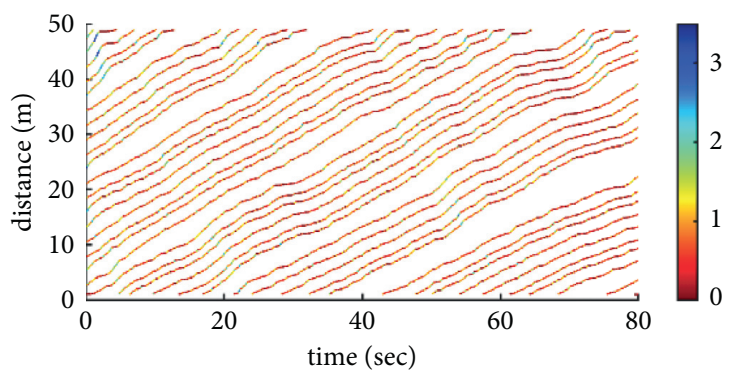

(e)

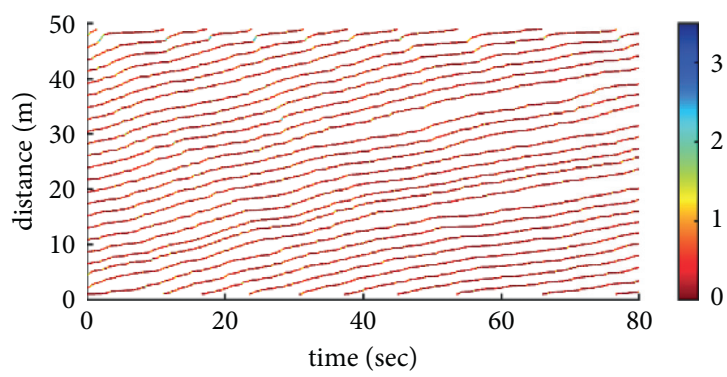

(g)

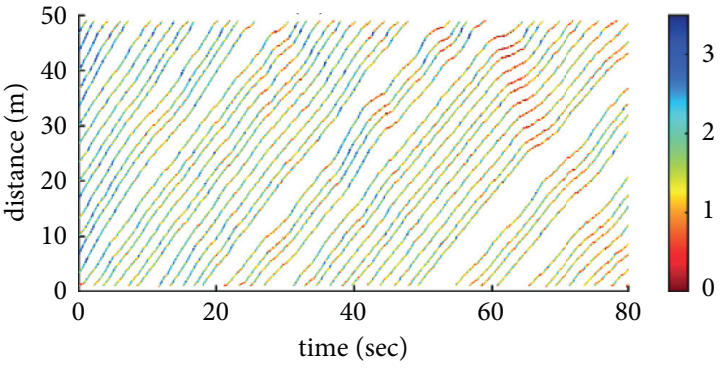

(b)

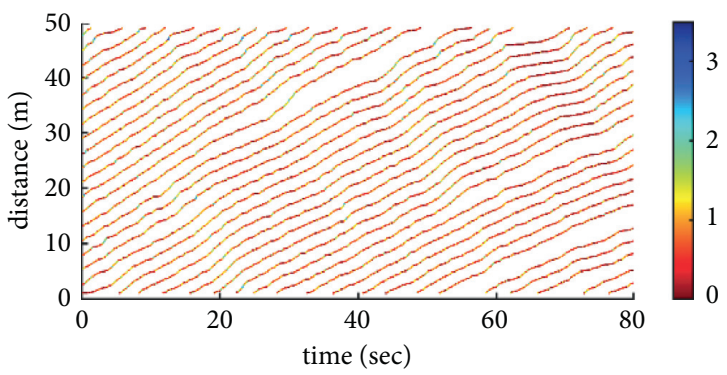

(d)

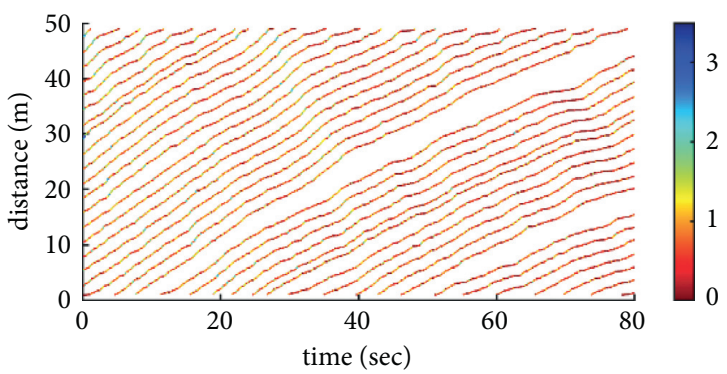

(f)

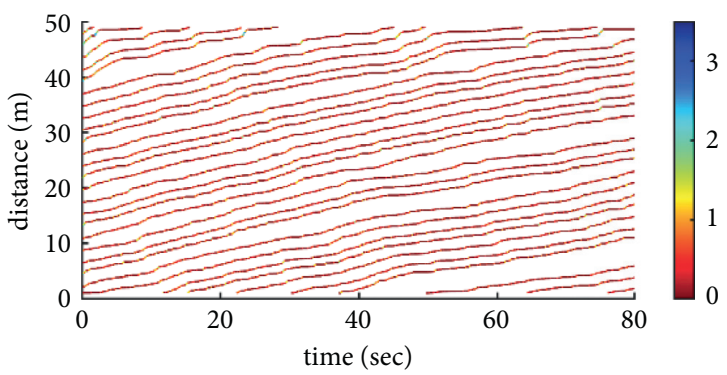

(h)

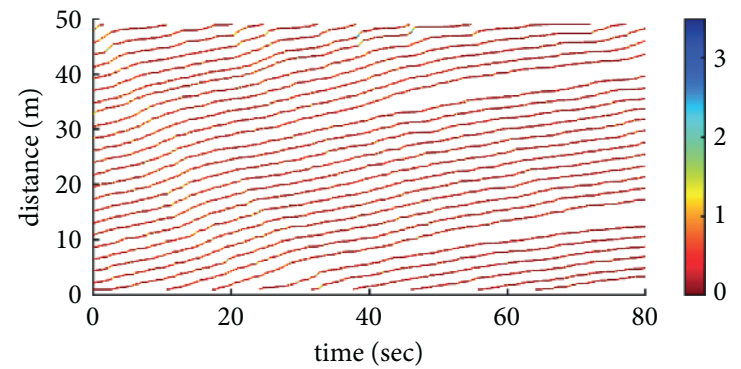

(i)

Figure 8: Each bicycle's trajectory in Case IV under different $N$. (a-c) Left lane, (d-f) middle lane, and (g-i) right lane. (a) $N=15$. (b) $N=15$. (c) $N=15$. (d) $N=19$. (e) $N=19$. (f) $N=19$. (g) $N=23$. (h) $N=23$. (i) $N=23$. 
TABLE 2: Calibrated parameters of the modified Newell model.

\begin{tabular}{|c|c|c|c|c|c|}
\hline Group mode & Group size & $\rho_{j}(\mathrm{bic} / \mathrm{km})$ & $\mu$ & $V_{f}(\mathrm{~km} / \mathrm{h})$ & RMSE \\
\hline No group & 1 & 638.7 & 4450 & 13.1 & 1.031 \\
\hline \multirow{2}{*}{ Shoulder group } & 2 & 606.2 & 3951 & 13.1 & 1.008 \\
\hline & 3 & 578 & 3818 & 9.184 & 0.6233 \\
\hline \multirow{2}{*}{ Following group } & 2 & 920.4 & 5100 & 12.97 & 0.823 \\
\hline & 3 & 717.6 & 7360 & 11.89 & 0.6728 \\
\hline
\end{tabular}

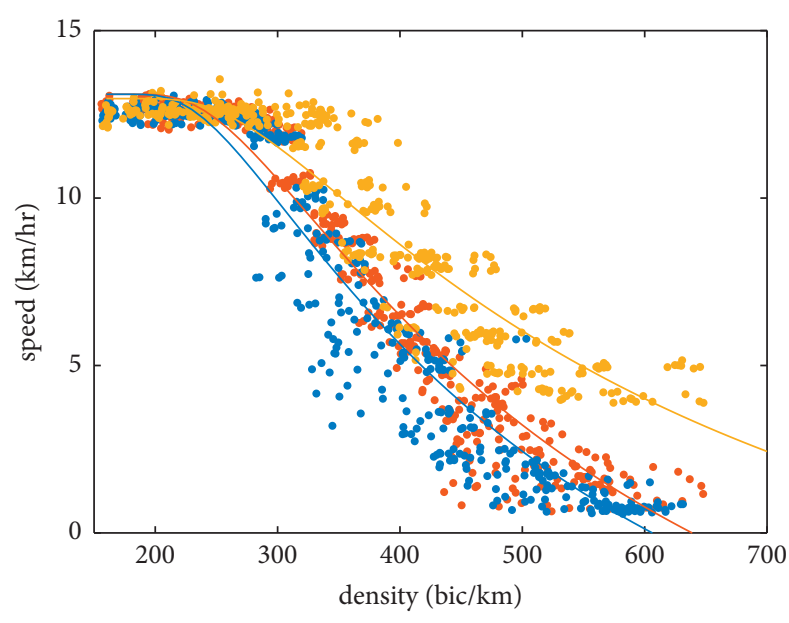

- without group behavior

- with shoulder group behavior

- with following group behavior

(a)

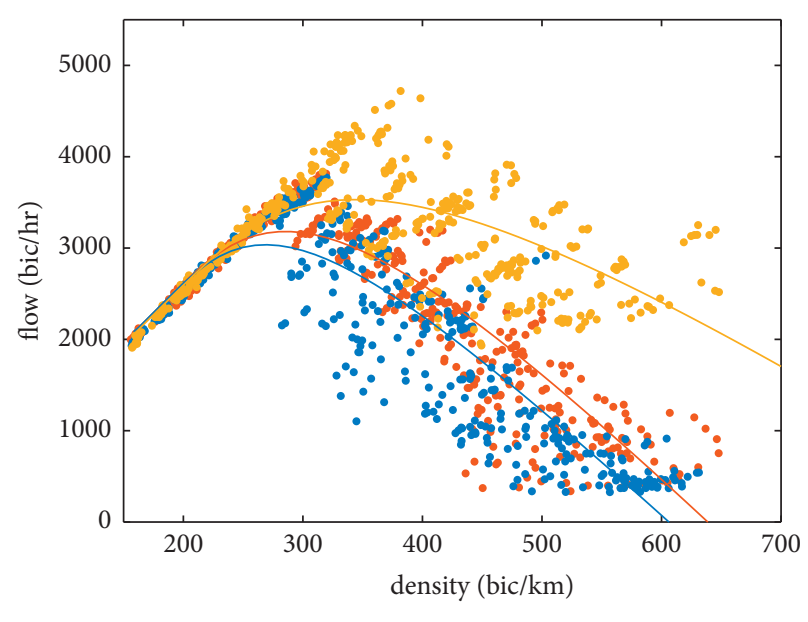

- without group behavior

- with shoulder group behavior

- with following group behavior

(b)

Figure 9: Fundamental diagrams under three kinds of bicycle flow. (a) Speed vs. density. (b) Flow vs. density.
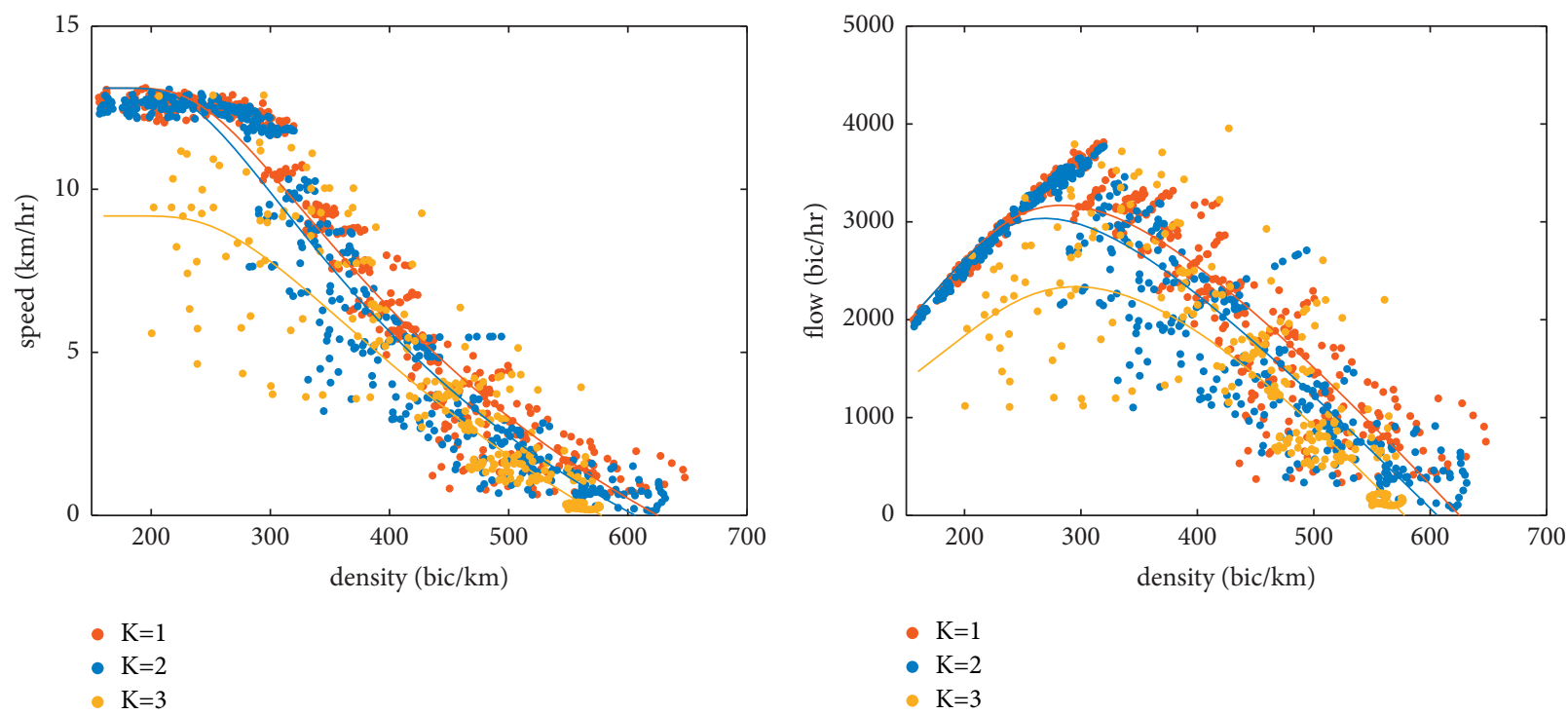

(a)

(b)

FIGURE 10: Fundamental diagrams under shoulder group behavior with different group sizes. (a) Speed vs. density. (b) Flow vs. density. 

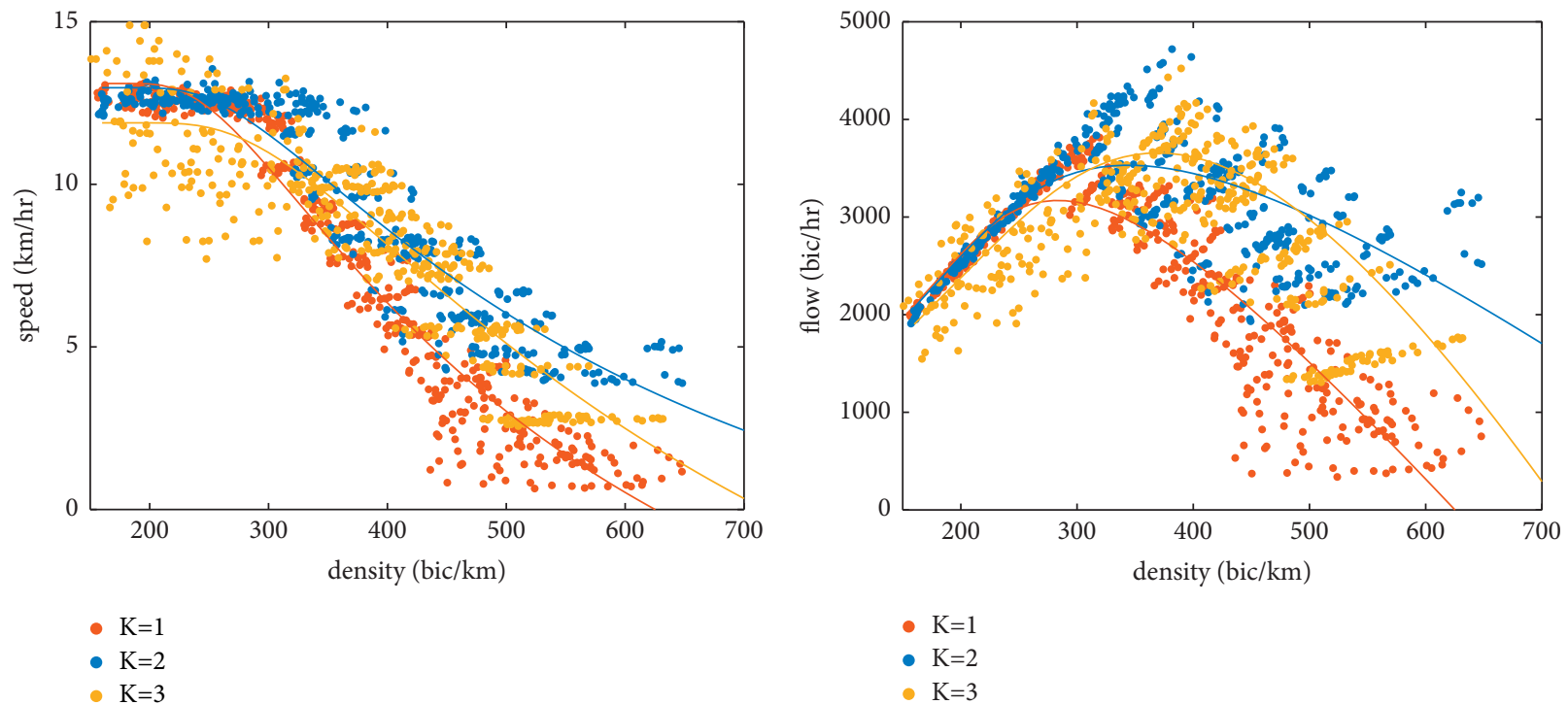

(a)

(b)

Figure 11: Fundamental diagrams under following group behavior with different group sizes. (a) Speed vs. density. (b) Flow vs. density.
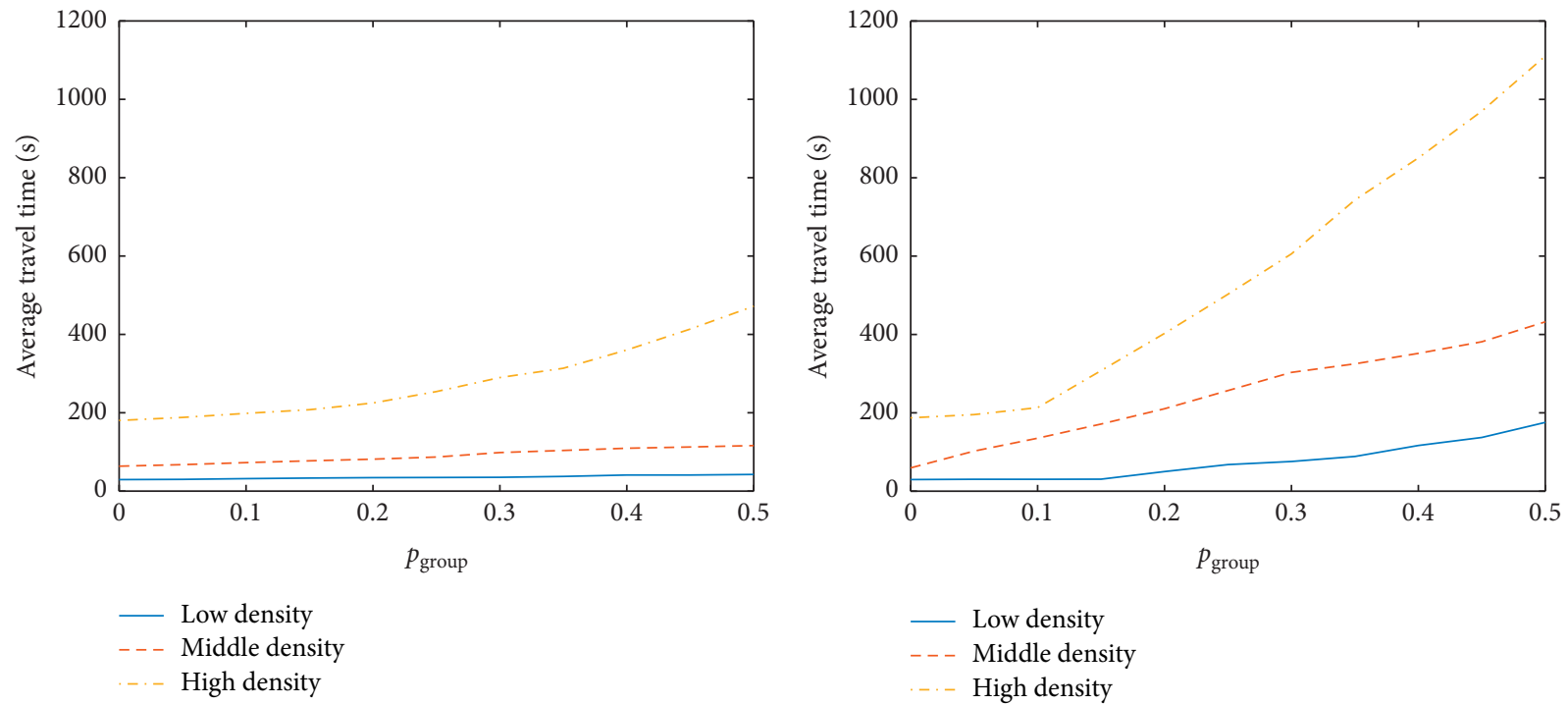

(a)

(b)

FIGURE 12: Continued. 


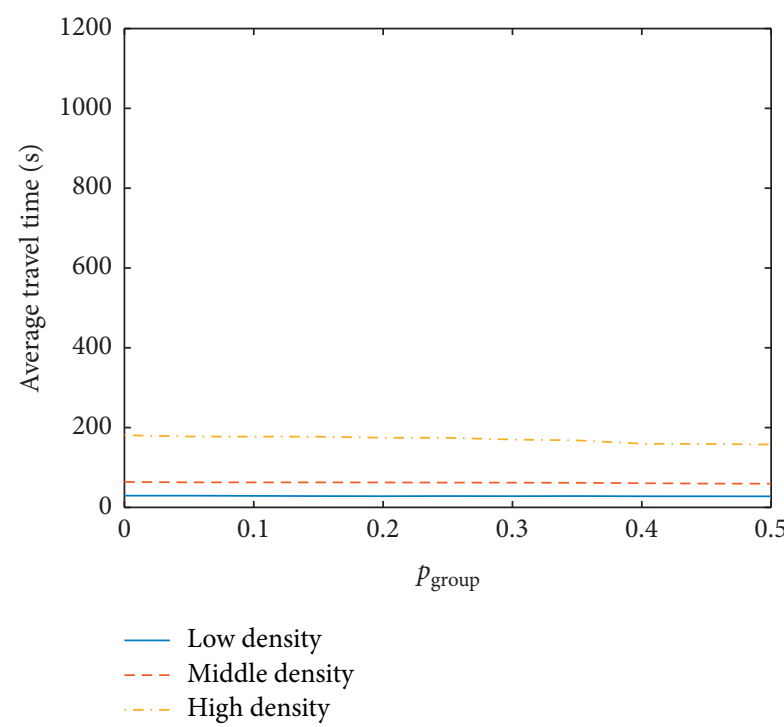

(c)

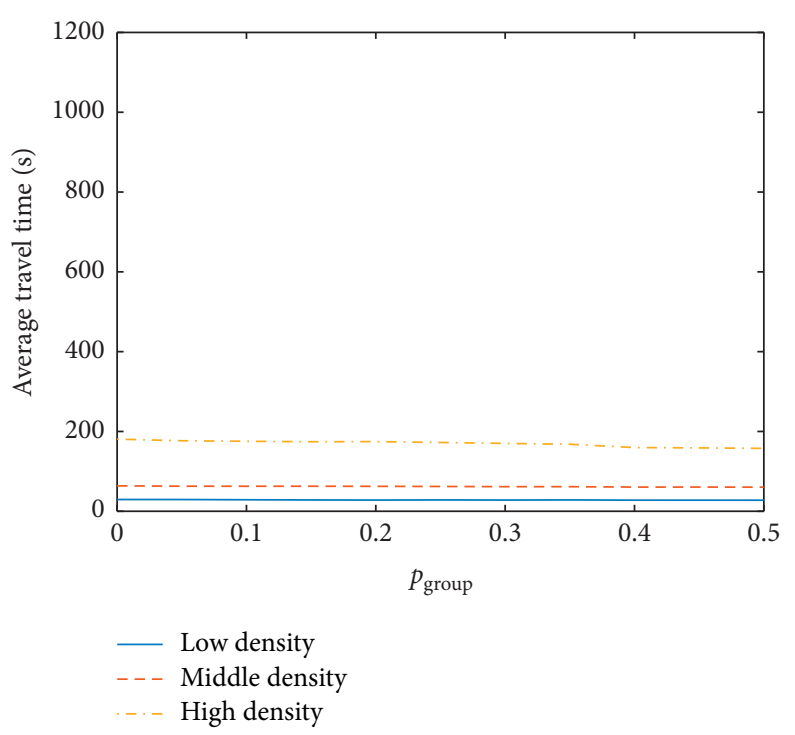

(d)

Figure 12: The average travel time under different group probabilities. (a, b) Shoulder group behavior and (c, (d) following group behavior. The group sizes are 2 in (a) and (c) and 3 in (b) and (d).

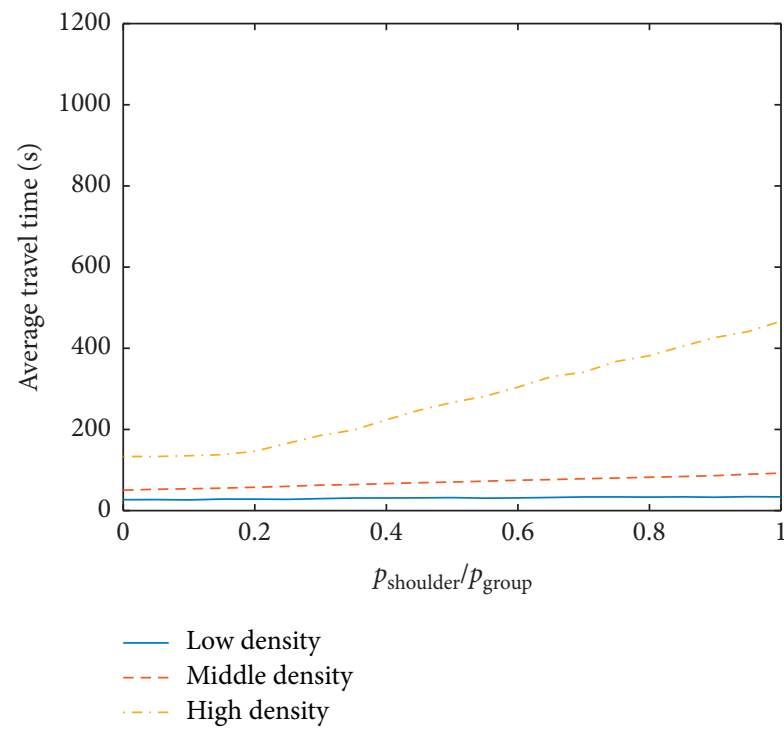

(a)

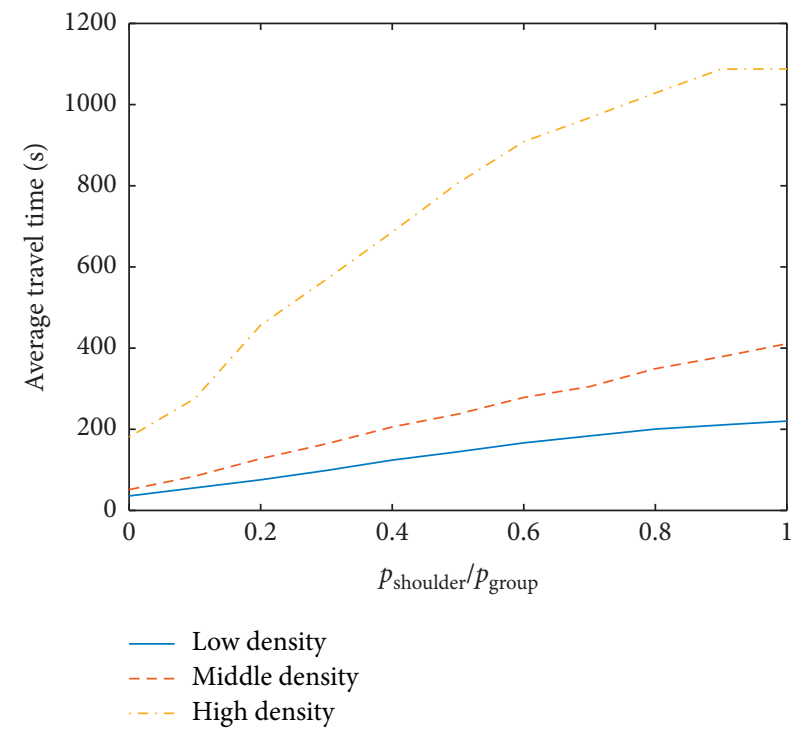

(b)

Figure 13: The average travel time under different shoulder group probabilities. The group sizes in (a) and (b) are, respectively, two and three.

impacts may be related to $p_{\text {group }}$. Thus, we apply the average travel time to evaluate the impacts of $p_{\text {group }}$ with different group sizes on bicycle flow, where the average travel time is defined as the average time of each bicycle's motion on the tested road. Figure 12 shows the average travel time under shoulder group behavior and following group behavior with different $p_{\text {group }}$ and different group sizes (i.e., $K=2$ or 3 ). From Figure 12, we can conclude the following findings:

(1) For shoulder group behavior, when $K=2$, $p_{\text {group }}$ has no prominent effects on the average travel time under low density and middle density but will prominently increase the average travel time under high density (see Figure 12(a)); when $K=3$, the average travel time always increases with $p_{\text {group }}$ (see Figure 12(b)). This shows that the negative impacts of shoulder group behavior become more prominent with the increase of $p_{\text {group }}$ and group size.

(2) For following group behavior, $p_{\text {group }}$ has no prominent impacts on the average travel time with different group sizes (see Figures 12(c) and 12(d)). 
In the analysis of Figure 12, we have evaluated the impacts of $p_{\text {group }}$ under two kinds of group behaviors on travel time. Then, we should explore the impacts of $p_{\text {group }}$ under coexistence of shoulder and following group behaviors on the travel time. Because coexistence of two kinds of group behavior is different from shoulder group behavior and following group behavior, we beforehand set the total group probability $p_{\text {group }}$ as 0.5 and make shoulder group probability $p_{\text {shoulder }}$ change from 0 to 0.5 . Thus, we can obtain the average travel time under coexistence of shoulder and following group behavior (see Figure 13). From Figure 13, we can conclude the following findings:

(1) When $K=2$, increasing the ratio of $p_{\text {shoulder }} / p_{\text {group }}$ has slight impacts on traffic efficiency under low or moderate density, while it has prominent negative impacts on traffic efficiency under high density, especially when the ratio is higher than 0.2 (see Figure 13(a)).

(2) When $K=3$, whether the density is low, moderate, or high, the increasing ratio has negative impacts on traffic efficiency, especially under the high density, and the impact is greater than that of $K=2$; e.g., when the ratio is 0.4 and density is high, the average travel time of $K=2$ and $K=3$ are, respectively, less than $300 \mathrm{~s}$ and more than $600 \mathrm{~s}$ (see Figures 13(a) and 13(b)).

(3) The above findings show that impacts of coexistence of shoulder and following group behavior are determined by density, group size, and ratio of $p_{\text {shoulder }} / p_{\text {group }}$. Thus, we recommend the administration reduce density, restrict group size, and decrease ratio of shoulder group behavior through diversion measures or prohibitions.

\section{Conclusions}

Many SF models have been developed to describe the bicycle's motion, but they cannot be used to directly study the impacts of group behavior on bicycle flow. In this paper, we first explore the motion features of bicycles with shoulder group behavior and following group behavior. Then, we propose an extended SF model for bicycle flow in groups. Numerical tests on one three-lane circuit road are conducted to explore the impacts of group behaviors on bicycle flow. The numerical results show that shoulder group behavior has some negative impacts on bicycle flow and should be prohibited; following group behavior has some positive impacts on bicycle flow and should be promoted; the impacts of coexistence of shoulder and following group behavior are related to the density. Besides, increasing group size, group probability, and density will enlarge the negative impacts of shoulder group behavior and alleviate the positive impacts of following group behavior.

However, some main parameters of the proposed model and the numerical results are not calibrated by experimental/ empirical data. Hence, we should in the future use some experimental/empirical data to further explore the quantitative impacts of group behavior on bicycle flow.

\section{Data Availability}

The data can be obtained from the corresponding author upon request.

\section{Conflicts of Interest}

The authors declare that they have no conflicts of interest.

\section{References}

[1] A. Gavriilidou, M. J. Wierbos, W. Daamen, Y. Yuan, V. L. Knoop, and S. P. Hoogendoorn, "Large-scale bicycle flow experiment: setup and implementation," Transportation Research Record: Journal of the Transportation Research Board, vol. 2673, no. 5, pp. 709-719, 2019.

[2] M. L. Zhang, "Analysis of factors influencing short-term demand for bike sharing," China Business and Trade, vol. 804, no. 5, pp. 109-110, 2020.

[3] B. C. Langford, J. Chen, and C. R. Cherry, "Risky riding: naturalistic methods comparing safety behavior from conventional bicycle riders and electric bike riders," Accident Analysis \& Prevention, vol. 82, pp. 220-226, 2015.

[4] Q. Liu, J. Sun, Y. Tian, Y. Ni, and S. Yu, "Modeling and simulation of nonmotorized vehicles' dispersion at mixed flow intersections," Journal of Advanced Transportation, vol. 2019, Article ID 9127062, 19 pages, 2019.

[5] K. Nagel and M. Schreckenberg, "A cellular automaton model for freeway traffic," Journal de Physique I, vol. 2, no. 12, pp. 2221-2229, 1992.

[6] H. Twaddle, T. Schendzielorz, and O. Fakler, "Bicycles in urban areas: review of existing methods for modeling behavior," Transportation Research Record: Journal of the Transportation Research Board, vol. 2434, no. 1, pp. 140-146, 2014.

[7] G. Gould and A. Karner, "Modeling bicycle facility operation: cellular automaton approach," Transportation Research Record: Journal of the Transportation Research Board, vol. 2140, no. 1, pp. 157-164, 2009.

[8] H. Ou, T.-Q. Tang, Y.-X. Rui, and J.-M. Zhou, "Modeling electric bicycle's abnormal behavior at a signalized intersection," Physica A: Statistical Mechanics and its Applications, vol. 511, pp. 218-231, 2018.

[9] G. Ren, H. Jiang, J. Chen, Z. Huang, and L. Lu, "Heterogeneous cellular automata model for straight-through bicycle traffic at signalized intersection," Physica A: Statistical Mechanics and its Applications, vol. 451, pp. 70-83, 2016.

[10] T.-Q. Tang, X.-F. Luo, J. Zhang, and L. Chen, "Modeling electric bicycle's lane-changing and retrograde behaviors," Physica A: Statistical Mechanics and its Applications, vol. 490, pp. 1377-1386, 2018.

[11] D. Helbing and P. Molnár, "Social force model for pedestrian dynamics," Physical Review, vol. 51, no. 5, pp. 4282-4286, 1995.

[12] D. Helbing, I. J. Farkas, and T. Vicsek, "Freezing by heating in a driven mesoscopic system," Physical Review Letters, vol. 84, no. 6, pp. 1240-1243, 2000.

[13] B. Anvari, M. G. H. Bell, A. Sivakumar, and W. Y. Ochieng, "Modelling shared space users via rule-based social force model," Transportation Research Part C: Emerging Technologies, vol. 51, pp. 83-103, 2015.

[14] Z. Ma, J. Xie, X. Qi, Y. Xu, and J. Sun, "Two-dimensional simulation of turning behavior in potential conflict area of 
mixed-flow Intersections," Computer-Aided Civil and Infrastructure Engineering, vol. 32, no. 5, pp. 412-428, 2017.

[15] D. Yang, X. Zhou, D. Wu, and S. Liu, "Study on the invasion behavior of e-bikes with motor vehicle traffic at a signalized intersection," Transportation Research Record: Journal of the Transportation Research Board, vol. 2672, no. 31, pp. 33-40, 2018.

[16] Y. Zhao and H. M. Zhang, "A unified follow-the-leader model for vehicle, bicycle and pedestrian traffic," Transportation Research Part B: Methodological, vol. 105, pp. 315-327, 2017.

[17] Y. Ni, Y. Li, X. Li, and J. Sun, "Modeling and simulation of the non-motorized traffic flow on physically separated bicycle roadways," Tongji Daxue Xuebao/Journal of Tongji University, vol. 47, no. 6, pp. 778-786, 2019.

[18] D. Helbing, D. Brockmann, T. Chadefaux et al., "Saving human lives: what complexity science and information systems can contribute," Journal of Statistical Physics, vol. 158, no. 3, pp. 735-781, 2015.

[19] M. Perc, "The social physics collective," Scientific Reports, vol. 9, no. 1, Article ID 16549, 2019.

[20] A. Gavriilidou, W. Daamen, Y. Yuan, and S. P. Hoogendoorn, "Modelling cyclist queue formation using a two-layer framework for operational cycling behaviour," Transportation Research Part C: Emerging Technologies, vol. 105, pp. 468-484, 2019.

[21] Y. X. Li, Y. Ni, and J. Sun, "A modified social force model for high-density through bicycle flow at mixed-traffic intersections," Simulation Modelling Practice and Theory, vol. 108, Article ID 102265, 2021.

[22] T.-Q. Tang, Y.-X. Rui, J. Zhang, and H.-Y. Shang, "A cellular automation model accounting for bicycle's group behavior," Physica A: Statistical Mechanics and its Applications, vol. 492, pp. 1782-1797, 2018.

[23] T.-Q. Tang, Y.-X. Rui, J. Zhang, and T. Wang, "Impacts of group behavior on bicycle flow at a signalized intersection," Physica A: Statistical Mechanics and its Applications, vol. 512, pp. 1205-1215, 2018.

[24] G. F. Newell, "Nonlinear effects in the dynamics of car following," Operations Research, vol. 9, no. 2, pp. 209-229, 1961. 